\title{
On exact controllability of neutral type time-delay systems
}

\section{K вопросу точной управляемости систем с запаздыванием нейтрального типа}

R. Rabah, IRCCyN, École des Mines de Nantes, 4 rue Alfred Kastler, BP 20722 Nantes, France. E-mail: rabah.rabah@mines-nantes.fr.

G. M. Sklyar, Institute of Mathematics, University of Szczecin and Karazin Kharkiv University, Wielkopolska 15, 70-451, Szczecin, Poland. E-mail: sklar@univ.szczecin.pl.

P. Yu. Barkhayev, Institute for Low Temperature Physics and Engineering, Academy of Sciences of Ukraine and Karazin Kharkiv University, 47 Lenin Ave., 61103 Kharkiv, Ukraine. E-mail: barkhayev@ilt.kharkov.ua.

\begin{abstract}
The paper is devoted to the problem of exact controllability for a wide class of neutral and mixed time-delay systems. We consider an equivalent operator model in Hilbert space and formulate steering conditions of controllable states as a vector moment problem. The existence of a basis of eigenvectors of the system operator enables the form of the moment problem to be substantially simplified. A change in control by a feedback law modifies the system structure to guarantee the existence of a basis of eigenvectors of the corresponding operator. We prove a criterion of exact controllability and ascertain the precise critical time of controllability.
\end{abstract}

\begin{abstract}
Анотація
Робота присвячена вирішенню задачі точної керованості для досить широкого класу систем з запізненням нейтрального та змішаного типів. Розглядаючи еквівалентну операторну модель в гільбертовому просторі, ми формулюємо умови керованості у вигляді деякої векторної проблеми моментів. Вид даної проблеми моментів істотно спрощується при наявності базису простору з власних векторів оператора системи з запізненням. Заміна керування дозволяе перетворити структуру системи, і гарантувати існування базису з власних векторів відповідного оператора. Ми доводимо критерій точної керованості і встановлюємо точний час керування.
\end{abstract}

\section{Аннотация}

Данная работа посвящена решению задачи точной управляемости для достаточно широкого класса систем с запаздыванием нейтрального и смешанного типов. Рассматривая эквивалентную операторную модель в гильбертовом пространстве, мы формулируем условия управляемости в виде некоторой векторной проблемы моментов. Вид данной проблемы моментов существенно упрощается при наличии базиса пространства из собственных векторов оператора системы с запаздыванием. Замена управления позволяет преобразовать структуру системы, и гарантировать существование базиса из собственных векторов соответствующего оператора. Мы доказываем критерий точной управляемости и устанавливаем точное время управления. 


\section{Introduction}

The controllability problem for linear time-delay systems has quite a long history (see, e.g. $[4,2,6,9,11,12]$ and references therein). In this paper we consider the problem of exact controllability for a large class of neutral type systems given by the following equation:

$$
\dot{z}(t)=A_{-1} \dot{z}(t-1)+L z_{t}+B u, \quad t \geq 0,
$$

where $A_{-1} \in \mathbb{R}^{n \times n}, B \in \mathbb{R}^{n \times r}$ are constant matrices, $z_{t}:[-1,0] \rightarrow \mathbb{C}^{n}$ is the history of $z$ defined by $z_{t}(s)=z(t+s)$, the delay operator $L$ is given by

$$
L f=\int_{-1}^{0} A_{2}(\theta) \frac{\mathrm{d}}{\mathrm{d} \theta} f(\theta) \mathrm{d} \theta+\int_{-1}^{0} A_{3}(\theta) f(\theta) \mathrm{d} \theta,
$$

and $A_{2}, A_{3}$ are $n \times n$-matrices whose elements belong to $L_{2}([-1,0], \mathbb{C})$; control function $u$ also belongs to $L_{2}\left(0, T ; \mathbb{C}^{r}\right)$.

The representation of delay systems as systems in some functional space has proved to be one of the most productive approaches. Namely, it is possible to associate the delay system with the following infinite-dimensional model:

$$
\dot{x}=\mathcal{A} x+\mathcal{B} u, \quad x \in H,
$$

where $H$ is a Hilbert space and the linear operator $\mathcal{A}$ is the generator of a $C_{0^{-}}$-semigroup.

For finite-dimensional linear control systems of the form (1.2), Kalman's controllability concept is well-known: the reachability set from zero at time $T$ coincides with the whole phase space $\left(\mathcal{R}_{T}=H\right)$ for some $T>0$. Moreover, if there are no constraints on control, then the controllability time $T$ may be chosen arbitrarily. However, if the phase space $H$ is infinitedimensional, then the described property does not hold, in general. For delay systems, the reachability set is always a subset of the domain $\mathcal{D}(\mathcal{A})$ of the operator $\mathcal{A}$, thus, it is natural to pose the question of reaching the whole set $\mathcal{D}(\mathcal{A})$. Besides, for delay systems the minimal controllability time cannot be arbitrarily small, which leads to the problem of finding this minimal time of transfer from 0 to an arbitrary state of $\mathcal{D}(\mathcal{A})$. The following criterion of exact controllability has been obtained by the co-authors of the present paper [14].

Theorem 1.1 The neutral type system (1.1) is exactly controllable if and only if the following conditions are verified:

(i) there are no $\lambda \in \mathbb{C}$ and $y \in \mathbb{C}^{n} \backslash\{0\}$, such that $\left(\Delta_{\mathcal{A}}(\lambda)\right)^{*} y=0$ and $B^{*} y=0$, where

$$
\Delta_{\mathcal{A}}(\lambda)=\lambda I-\lambda \mathrm{e}^{-\lambda} A_{-1}-\lambda \int_{-1}^{0} \mathrm{e}^{\lambda s} A_{2}(s) \mathrm{d} s-\int_{-1}^{0} \mathrm{e}^{\lambda s} A_{3}(s) \mathrm{d} s,
$$

or equivalently, $\operatorname{rank}\left(\Delta_{\mathcal{A}}(\lambda) B\right)=n$ for any $\lambda \in \mathbb{C}$.

(ii) there are no $\mu \in \sigma\left(A_{-1}\right)$ and $y \in \mathbb{C}^{n} \backslash\{0\}$, such that $A_{-1}^{*} y=\bar{\mu} y$ and $B^{*} y=0$, or equivalently, $\operatorname{rank}\left(B \quad A_{-1} B \cdots A_{-1}^{n-1} B\right)=n$.

Moreover, if the conditions (i) and (ii) hold, then the system is exactly controllable at any time $T>n_{1}$ and not exactly controllable at any time $T \leq n_{1}$, where $n_{1}$ is the first controllability index of the pair $\left(A_{-1}, B\right)$.

If the maximal delay is equal to $h$, then the critical time of controllability is $T=n_{1} h$. 
We note that (1.1) is a system with distributed delay for which, in contrast to systems with several discrete delays (see $[2,9,3,10,13,20]$ and references therein), the explicit form of the semigroup is unknown, in general, which makes the analysis much more complicated. We also note, that an important advantage of the theorem is that it gives the exact critical time of controllability.

Besides, we can note that for linear retarded systems $\left(A_{-1}=0\right)$, the conditions of exact controllability imply $\operatorname{rank} B=n$, which is a very strong condition. This means that exact controllability is more typical for neutral type systems.

To study the exact controllability we use the moment problem approach: steering conditions of controllable states are represented as a vectorial trigonometric moment problem with respect to a special Riesz basis. We analyze the solvability of the non-Fourier moment problem obtained using methods developed in [1] (see also [24]).

The existence of a basis of the state space consisting of eigenvectors (or generalized eigenvectors) essentially simplifies the expression of the moment problem (see [18] and [22]). In our case, the existence of a basis of eigenvectors is determined by the form of the matrix $A_{-1}$ of the neutral term of the system (1.1), and, in general, such basis does not exist (see [15, 16]). This makes the procedure of the choice of a Riesz basis and further manipulations with it quite sophisticated in the general case $([14])$.

However, by means of a change in control in the initial system, it is possible to pass to an equivalent controllability problem for a system with a matrix $A_{-1}$ of simple structure. This structure guarantees the existence of a Riesz basis of eigenvectors for the state space. The form of the corresponding moment problem becomes simpler, which makes the constructions and the proofs of the main results both clear and illustrative.

In this paper, we provide proof of Theorem 1.1 for the system (1.1) with $A_{-1}$ of a special form and show that this fact implies the proof for a system with an arbitrary matrix $A_{-1}$. Besides, we consider the controllability problem for so-called mixed retarded-neutral type systems (see also [19]), which was considered in [14], and prove that if the neutral term is singular (det $\left.A_{-1}=0\right)$ and the pair $\left(A_{-1}, B\right)$ is uncontrollable, then the system (1.1) is uncontrollable as well.

The paper is organized as follows. In Section 2, we introduce the abstract equation and discuss how we can consider, without loss of generality, that the system has a special form with a Riesz basis of eigenvectors. In Section 3, using spectral Riesz bases, we represent the steering conditions as a vectorial moment problem. Section 4 is devoted to proving the necessity of controllability conditions and in Sections 5 and 6 we prove the sufficiency of these conditions for the cases of one-dimensional and multi-dimensional controls. Finally, in Section 7 we give an example illustrating the obtained results.

\section{Equivalent systems}

We consider the operator model of time-delay systems introduced in [5] (see also [8]). The state space is $M_{2}\left(-1,0 ; \mathbb{C}^{n}\right)=\mathbb{C}^{n} \times L_{2}\left(-1,0 ; \mathbb{C}^{n}\right)$, in short $M_{2}$, and the equation (1.1) may be rewritten in the following form:

$$
\dot{x}(t)=\mathcal{A} x(t)+\mathcal{B} u(t), \quad \mathcal{A}=\left(\begin{array}{cc}
0 & L \\
0 & \frac{\mathrm{d}}{\mathrm{d} \theta}
\end{array}\right), \mathcal{B}=\left(\begin{array}{c}
B \\
0
\end{array}\right),
$$

where the domain of the operator $\mathcal{A}$ is

$$
\mathcal{D}(\mathcal{A})=\left\{(y, z(\cdot)) \in M_{2}: z \in H^{1}\left(-1,0 ; \mathbb{C}^{n}\right), y=z(0)-A_{-1} z(-1)\right\}
$$


and $H^{1}$ is the Sobolev space of functions with derivative from $L_{2}$.

The reachability set from the initial state 0 at time $T$ is defined by

$$
\mathcal{R}_{T}=\left\{x: x=\int_{0}^{T} \mathrm{e}^{\mathcal{A} t} \mathcal{B} u(t) \mathrm{d} t, \quad u(\cdot) \in L_{2}\left(0, T ; \mathbb{C}^{r}\right)\right\}
$$

Further it is shown that $\mathcal{R}_{T} \subset \mathcal{D}(\mathcal{A})$ for all $T>0$.

Definition 2.1 We say that the system (2.4) is exactly controllable from zero by controls from $L_{2}$, if there is a time $T_{0}$ (critical time), such that for all $T>T_{0}$ one has

$$
\mathcal{R}_{T}=\mathcal{D}(\mathcal{A})
$$

and for all $T<T_{0}: \mathcal{R}_{T} \neq \mathcal{D}(\mathcal{A})$.

The given definition means that for some $T>0$ the set of solutions $\{z(t): t \in[T-1, T]\}$ of the system (1.1) coincides with space $H^{1}\left(T-1, T ; \mathbb{C}^{n}\right)$.

Lemma 2.2 If the system (1.1) is exactly controllable at time $T$, then for any matrix $P \in \mathbb{C}^{n \times r}$ the perturbed system

$$
\dot{z}(t)=\left(A_{-1}+B P\right) \dot{z}(t-1)+L z_{t}+B u
$$

is exactly controllable at the same time $T$.

Proof. Assume that the system(1.1) is controllable at time $T$. This means that for any function $f(t) \in H^{1}\left(T-1, T ; \mathbb{C}^{n}\right)$ there is a control $u(t) \in L_{2}\left(0, T ; \mathbb{C}^{n}\right)$, such that the solution of the equation

$$
\dot{z}(t)=A_{-1} \dot{z}(t-1)+L z_{t}+B u(t),
$$

with the initial condition $z(t)=0, t \in[-1,0]$ satisfies the relation $z(t)=f(t), t \in[T-1, T]$. Let us rewrite (2.6) in the form

$$
\dot{z}(t)=\left(A_{-1}+B P\right) \dot{z}(t-1)+L z_{t}+B v(t),
$$

where $v(t)=u(t)-P \dot{z}(t-1), t \in[0, T]$. Since $z(t-1) \in H^{1}\left(0, T ; \mathbb{C}^{n}\right)$, then $v(t) \in L_{2}\left(0, T ; \mathbb{C}^{n}\right)$. Therefore, the control $v(t)$ transfers the state $z(t)=0, t \in[-1,0]$ to the state $z(t)=f(t)$, $t \in[T-1, T]$ by virtue of the system (2.5). This means that (2.5) is also exactly controllable at the time $T$.

We have also equivalence in the conditions of exact controllability in Theorem 1.1.

Lemma 2.3 If the system (1.1) satisfies the conditions (i) and (ii) of Theorem 1.1, then a perturbed system (2.5) with an arbitrary matrix $P$ satisfies the same conditions.

The proof follows from the relation $\Delta_{\widehat{\mathcal{A}}}^{*}(\lambda) y=\left[\Delta_{\mathcal{A}}^{*}(\lambda)-\lambda \mathrm{e}^{-\lambda} P^{*} B^{*}\right] y=0$, where $\widehat{\mathcal{A}}$ is the operator corresponding to the system $(2.5)$, and from the fact that the property of controllability of a pair $(A, B)$ is invariant with respect to feedback changes in control (see, e.g. [23]).

Corollary 2.4 Therefore, if we prove Theorem 1.1 for the system (1.1) with a pair $\left(A_{-1}, B\right)$, then we also prove this theorem for all systems with a pair of matrices $\left(\widehat{A}_{-1}, B\right)$, where $\widehat{A}_{-1}=$ $A_{-1}+B P$. 
If the pair $(A, B)$ is controllable, then (see, e.g. [23]) for any set $S=\left\{\mu_{1}, \ldots, \mu_{n}\right\} \subset \mathbb{C}$, there is matrix $P \in \mathbb{C}^{r \times n}$ such that the set $S$ is the spectrum of $\sigma(A+B P)=S$. Thus, if we fix $n$ distinct real numbers

$$
\left\{\mu_{1}, \ldots, \mu_{n}\right\} \subset \mathbb{R}, \quad \mu_{i} \neq \mu_{j}, i \neq j, \quad \mu_{i} \notin\{0,1\},
$$

we can find a change in control $u(t)=P \dot{z}(t-1)+v(t), P \in \mathbb{C}^{r \times n}$, and a transformation of the state $z=C w$, which reduce the system to the following form

$$
\dot{w}(t)=\widehat{A}_{-1} \dot{w}(t-1)+\int_{-1}^{0} \widehat{A}_{2}(\theta) \dot{w}(t+\theta) \mathrm{d} \theta+\int_{-1}^{0} \widehat{A}_{3}(\theta) w(t+\theta) \mathrm{d} \theta+\widehat{B} v,
$$

where $\widehat{A}_{-1}=C^{-1}\left(A_{-1}+B P\right) C, \widehat{A}_{i}(\theta)=C^{-1} A_{i}(\theta) C, \widehat{B}=C^{-1} B$, satisfy the following conditions:

(a) the spectrum of $\widehat{A}_{-1}$ is $\sigma\left(\widehat{A}_{-1}\right)=\left\{\mu_{m}\right\}_{m=1}^{n}$;

(b) the pair $\left(\widehat{A}_{-1}, \widehat{B}\right)$ is in the Frobenius normal form (see [23]), i.e.

$$
\widehat{A}_{-1}=\operatorname{diag}\left\{F_{1}, \ldots, F_{r}\right\}, F_{i}=\left(\begin{array}{ccccc}
0 & 1 & 0 & \cdots & 0 \\
0 & 0 & 1 & \cdots & 0 \\
\vdots & \vdots & \vdots & \ddots & \vdots \\
0 & 0 & 0 & \cdots & 1 \\
a_{1}^{i} & a_{2}^{i} & a_{3}^{i} & \cdots & a_{s_{i}}^{i}
\end{array}\right)
$$

and $\widehat{B}=\operatorname{diag}\left\{g_{1}, \ldots, g_{r}\right\}$, where $g_{i}=(0,0, \ldots, 1)^{\mathrm{T}} \in \mathbb{C}^{s_{i}}$.

From these considerations we obtain the following lemma.

Lemma 2.5 The proof of sufficiency of Theorem 1.1 for the family of systems (2.8), verifying conditions (a)-(b), implies the sufficiency of these condition for arbitrary systems of type (1.1).

Remark 2.6 In the proof of Theorem 1.1 for the case of one-dimensional control $(r=1)$ it is enough to assume only condition (a). However, in the proof of the general case (multidimensional control), both conditions (a) and (b) are needed.

In the paper [14] the necessity of condition (ii) is proved with the assumption that the matrix $A_{-1}$ is non-singular. In the present paper, we complete the proof: if the pair $\left(A_{-1}, B\right)$ is not controllable, then the system (1.1) is also not controllable (Theorem 4.6).

Furthermore, without loss of generality, we may assume that the conditions (a) and (b) hold for the pair $\left(A_{-1}, B\right)$. Due to this construction, we have $\operatorname{det} A_{-1} \neq 0$ and we denote by $\left\{c_{m}\right\}_{m=1}^{n}$ the basis of the normed eigenvectors of $A_{-1}$.

\section{Riesz basis and the moment problem}

Let us denote by $\widetilde{\mathcal{A}}$ the operator $\mathcal{A}$ in the case $A_{2}(\theta)=A_{3}(\theta) \equiv 0$. The eigenvalues of $\widetilde{\mathcal{A}}$ are of the form (see $[16])$ :

$$
\sigma(\widetilde{\mathcal{A}})=\left\{\widetilde{\lambda}_{m}^{k}=\ln \left|\mu_{m}\right|+2 k \pi \mathrm{i}, m=1, \ldots, n, k \in \mathbb{Z}\right\} \cup\{0\},
$$


where $\left\{\mu_{1}, \ldots, \mu_{n}\right\}=\sigma\left(A_{-1}\right)$. Since all eigenvalues of $A_{-1}$ are simple, then the operator $\widetilde{\mathcal{A}}$ possesses simple eigenvalues only, and only one eigenvector $\widetilde{\varphi}_{m, k}=\left(0, \mathrm{e}^{\widetilde{\lambda}_{m}^{t}} c_{m}\right)^{T}$ corresponds to each eigenvalue $\widetilde{\lambda}_{m}^{k}$ and there are no root-vectors. Moreover, the following estimates hold

$$
0<\inf _{k \in \mathbb{Z}}\left\|\widetilde{\varphi}_{m, k}\right\| \leq \sup _{k \in \mathbb{Z}}\left\|\widetilde{\varphi}_{m, k}\right\|<+\infty .
$$

The spectrum of $\mathcal{A}$ is of the following form (see [16]):

$$
\sigma(\mathcal{A})=\left\{\ln \left|\mu_{m}\right|+2 k \pi \mathrm{i}+O(1 / k), m=1, \ldots, n, k \in \mathbb{Z}\right\} .
$$

There exists $N \in \mathbb{N}$ such that for all $m=1, \ldots, n$ and for all $k:|k|>N$ the total multiplicity of the eigenvalues of $\mathcal{A}$, contained in the circles $L_{m}^{k}\left(r^{(k)}\right)$ equals 1 , where $L_{m}^{k}\left(r^{(k)}\right)=L_{m}^{k}$ are circles with radii $r^{(k)}$ centered at $\widetilde{\lambda}_{m}^{k}$, and the relation $\sum_{k \in \mathbb{Z}}\left(r^{(k)}\right)^{2}<\infty$ is satisfied ([17, Theorem 4]). We denote these eigenvalues of the operator $\mathcal{A}$ as $\lambda_{m}^{k}$ and the corresponding eigenvectors as $\varphi_{m, k}, m=1, \ldots, n,|k|>N$.

Assume that the vectors $\varphi_{m, k}$ are normed such that $P_{m}^{(k)} \widetilde{\varphi}_{m, k}=\varphi_{m, k}$, where $P_{m}^{(k)}=$ $\frac{1}{2 \pi \mathrm{i}} \int_{L_{m}^{(k)}} R(\lambda, \mathcal{A}) \mathrm{d} \lambda$ are spectral projectors and $R(\lambda, \mathcal{A})$ is the resolvent of $\mathcal{A}$.

The families $\left\{\varphi_{m, k}\right\}$ and $\left\{\widetilde{\varphi}_{m, k}\right\}$ are quadratically close: $\sum_{|k|>N} \sum_{m=1}^{n}\left\|\varphi_{m, k}-\widetilde{\varphi}_{m, k}\right\|^{2}<\infty$, which, in particular, implies the following estimates

$$
0<\inf _{|k|>N}\left\|\varphi_{m, k}\right\| \leq \sup _{|k|>N}\left\|\varphi_{m, k}\right\|<+\infty .
$$

The explicit form of eigenvectors of $\mathcal{A}$ is $\varphi_{m, k}=\left(\left(I-\mathrm{e}^{\lambda_{m}^{k}} A_{-1}\right) x_{m, k}, \mathrm{e}^{\lambda_{m}^{k} \theta} x_{m, k}\right)^{T}$, where $x_{m, k} \in$ $\operatorname{Ker} \Delta_{\mathcal{A}}\left(\lambda_{m}^{k}\right)$.

Outside the circles $L_{m}^{k},|k|>N, m=1, \ldots, n$, there is only a finite number of eigenvalues of $\mathcal{A}$, which we denote by $\widehat{\lambda}_{s}, s=1, \ldots, \ell_{N}$ counted with multiplicities. We denote by $\widehat{\varphi}_{s}$ the corresponding generalized eigenvectors of the operator $\mathcal{A}$. The family

$$
\{\varphi\}=\left\{\varphi_{m, k}\right\} \cup\left\{\widehat{\varphi}_{s}\right\}
$$

forms a Riesz basis of the space $M_{2}([16])$.

We denote by

$$
\{\psi\}=\left\{\psi_{m, k}\right\} \cup\left\{\widehat{\psi}_{s}\right\}
$$

the family of eigenvectors of the adjoint operator $\mathcal{A}^{*}$, which is biorthogonal to $\{\varphi\}$. Here $\mathcal{A}^{*} \psi_{m, k}=\overline{\lambda_{m}^{k}} \psi_{m, k}, m=1, \ldots, n,|k|>N$. The explicit form of the eigenvectors of the adjoint operator $\mathcal{A}^{*}$ is

$$
\psi_{m, k}=\left(y_{m, k},\left[\overline{\lambda_{m}^{k}} \mathrm{e}^{-\overline{\lambda_{m}^{k}} \theta} I-A_{2}^{*}(\theta)+\int_{0}^{\theta} \mathrm{e}^{\overline{\lambda_{m}^{k}}(s-\theta)}\left(A_{3}^{*}(s)+\overline{\lambda_{m}^{k}} A_{2}^{*}(s)\right) \mathrm{d} s\right] y_{m, k}\right)^{T},
$$

where $y_{m, k} \in \operatorname{Ker} \Delta_{\mathcal{A}}^{*}\left(\overline{\lambda_{m}^{k}}\right)$.

The family (3.12) forms a Riesz basis of the space $M_{2}$. The proofs of the propositions mentioned in this section may be found in $[15,16,17]$.

Let us pose the controllability problem as a moment problem. To do this, we expand the steering condition $x_{T}=\left(\begin{array}{c}y_{T} \\ z_{T}(\cdot)\end{array}\right)=\int_{0}^{T} \mathrm{e}^{\mathcal{A} t} \mathcal{B} u(t) \mathrm{d} t$ with respect to the biorthogonal bases $\{\varphi\}$ 
and $\{\psi\}$ given by (3.11) and (3.12). A state $x_{T} \in M_{2}$ is reachable at a time $T$ if and only if

$$
\sum_{\varphi \in\{\varphi\}}\left\langle x_{T}, \psi\right\rangle \varphi=\sum_{\varphi \in\{\varphi\}} \int_{0}^{T}\left\langle\mathrm{e}^{\mathcal{A} t} \mathcal{B} u(t), \psi\right\rangle \mathrm{d} t \cdot \varphi .
$$

Here, we denote by $\langle\cdot, \cdot\rangle$ the scalar product in the space $M_{2}:\langle\cdot, \cdot\rangle_{M_{2}}$.

Let $\left\{b_{1}, \ldots, b_{r}\right\}$ be an arbitrary basis of the image of the matrix $B$ and $\mathbf{b}_{d}=\left(b_{d}, 0\right)^{T} \in M_{2}$, $d=1, \ldots, r$. Then the steering condition is equivalent to the following system of equalities:

$$
\begin{aligned}
\left\langle x_{T}, \psi\right\rangle & =\int_{0}^{T}\left\langle\mathrm{e}^{\mathcal{A} t} \mathcal{B} u(t), \psi\right\rangle \mathrm{d} t \\
& =\sum_{d=1}^{r} \int_{0}^{T}\left\langle\mathrm{e}^{\mathcal{A} t} \mathbf{b}_{d}, \psi\right\rangle u_{d}(t) \mathrm{d} t,
\end{aligned}
$$

where $\psi \in\{\psi\}, u(\cdot) \in L_{2}\left(0, T ; \mathbb{C}^{r}\right)$. Using the representation (3.13) for eigenvectors $\psi=\psi_{m, k}$, $m=1, \ldots, n,|k|>N$, we obtain the following identity:

$$
\left\langle\mathrm{e}^{\mathcal{A} t} \mathbf{b}_{d}, \psi_{m, k}\right\rangle_{M_{2}}=\mathrm{e}^{\lambda_{m}^{k} t}\left\langle\mathbf{b}_{d}, \psi_{m, k}\right\rangle_{M_{2}}=\mathrm{e}^{\lambda_{m}^{k} t}\left\langle b_{d}, y_{m, k}\right\rangle_{\mathbb{C}^{n}},
$$

where $y_{m, k} \in \operatorname{Ker} \Delta_{\mathcal{A}}^{*}\left(\overline{\lambda_{m}^{k}}\right)$. Let us introduce the notation:

$$
q_{m, k}^{d}=k\left\langle\mathbf{b}_{d}, \psi_{m, k}\right\rangle_{M_{2}} .
$$

Due to (3.15), the equalities (3.14) corresponding to $\psi \in\left\{\psi_{m, k},|k|>N, m=1, \ldots, n\right\}$ take the form:

$$
k\left\langle x_{T}, \psi_{m, k}\right\rangle=\sum_{d=1}^{r} \int_{0}^{T} \mathrm{e}^{\lambda_{m}^{k} t} q_{m, k}^{d} u_{d}(t) \mathrm{d} t .
$$

Besides, for generalized eigenvectors $\psi=\widehat{\psi}_{s}, s=1, \ldots, \ell_{N}$, the following relations hold:

$$
\left\langle\mathrm{e}^{\mathcal{A} t} \mathbf{b}_{d}, \psi\right\rangle,=\left\langle\mathbf{b}_{d}, \mathrm{e}^{\mathcal{A}^{*} t} \psi\right\rangle=\widehat{q}_{s}^{d}(t) \mathrm{e}^{\widehat{\lambda}_{s} t},
$$

where $\widehat{q}_{s}^{d}(t)$ are polynomials of appropriate degrees. Therefore, the equalities (3.14) corresponding to $\psi \in\left\{\widehat{\psi}_{s}\right\}$ take the form:

$$
\left\langle x_{T}, \widehat{\psi}_{s}\right\rangle=\sum_{d=1}^{r} \int_{0}^{T} \mathrm{e}^{\widehat{\lambda}_{s} t} \widehat{q}_{s}^{d}(t) u_{d}(t) \mathrm{d} t .
$$

Thus, a state $x_{T} \in M_{2}$ is reachable from 0 at time $T>0$ if and only if for some controls $u_{d}(\cdot) \in L_{2}(0, T), d=1, \ldots, r$ the equalities (3.17) and (3.18) hold.

The moment problem obtained (3.17)-(3.18) is the main object of our further analysis. We conclude this section by two estimates which are important for the further analysis.

Lemma 3.1 There is a constant $\delta_{1}>0$ such that

$$
\left|q_{m, k}^{d}\right| \leq \delta_{1}, \quad m=1, \ldots, n,|k|>N, d=1, \ldots, r .
$$

Lemma 3.2 There is a sequence $\left\{\alpha_{k}\right\}, \sum_{|k|>N} \alpha_{k}^{2}<+\infty$, such that for all $m=1, \ldots, n,|k|>N$, $d=1, \ldots, r$ and $t \in[0, T]$ the following estimate holds:

$$
\left|\mathrm{e}^{\lambda_{m}^{k} t}\left\langle\mathbf{b}_{d}, \psi_{m, k}\right\rangle_{M_{2}}-\mathrm{e}^{\tilde{\lambda}_{m}^{k} t}\left\langle\mathbf{b}_{d}, \widetilde{\psi}_{m, k}\right\rangle_{M_{2}}\right| \leq \frac{\alpha_{k}}{|k|} .
$$

The proofs of these propositions may be found in [14]. 


\section{Necessary conditions of controllability}

Let us investigate the solvability of the equations (3.17)-(3.18). The following well-known result is a consequence of the Bari theorem (see [7],[24]).

Lemma 4.1 Consider the following moment problem:

$$
s_{k}=\int_{0}^{T} g_{k}(t) u(t) \mathrm{d} t, \quad T>0, k \in \mathbb{N}
$$

where $g_{k}(\cdot) \in L_{2}(0, T)$ for all $k \in \mathbb{N}$. The following statements are equivalent:

(i) For the sequence $\left\{s_{k}\right\}_{k \in \mathbb{N}}$ the problem (4.21) has a solution $u(\cdot) \in L_{2}(0, T)$ if and only if $\left\{s_{k}\right\} \in \ell_{2}$, i.e. $\sum_{k \in \mathbb{N}} s_{k}^{2}<+\infty$;

(ii) the family $\left\{g_{k}(t)\right\}_{k \in \mathbb{N}}, t \in[0, T]$ forms a Riesz basis in the closure of its linear span

$$
\mathrm{Cl} \operatorname{Lin}\left\{g_{k}(t), k \in \mathbb{N}\right\} .
$$

The following propositions on the solvability of the moment problem were proved in [14].

Lemma 4.2 Let us suppose that for some $T_{1}>0$ the functions $\left\{g_{k}(t)\right\}_{k \in \mathbb{N}}$, defined on $\left[0, T_{1}\right]$, form a Riesz basis in $\mathrm{Cl} \operatorname{Lin}\left\{g_{k}(t), k \in \mathbb{N}\right\} \subset L_{2}\left(0, T_{1}\right)$ and codim $\mathrm{Cl} \operatorname{Lin}\left\{g_{k}(t), k \in \mathbb{N}\right\}<+\infty$. Then for any $T: 0<T<T_{1}$, there is an infinite-dimensional subspace $\ell_{T} \subset \ell_{2}$, such that the moment problem (4.21) is unsolvable on $[0, T]$ for $\left\{s_{k}\right\} \in \ell_{T} \backslash\{0\}$.

Lemma 4.3 Let us consider the moment problem

$$
s_{k}=\sum_{d=1}^{r} \int_{0}^{T} g_{k}^{d}(t) u_{d}(t) \mathrm{d} t, \quad k \in \mathbb{N},
$$

assuming that $\sum_{k \in \mathbb{N}} \int_{0}^{T}\left|g_{k}^{d}(t)\right|^{2} \mathrm{~d} t<+\infty$ for all $d=1, \ldots, r$.

Then the set $S_{0, T}$ of sequences $\left\{s_{k}\right\}$ for which the problem (4.22) is solvable is a nontrivial submanifold of $\ell_{2}$, i.e. $S_{0, T} \neq \ell_{2}$.

The following proposition (see [14]) shows that the reachability set $\mathcal{R}_{T}$ is always a subset of $\mathcal{D}(\mathcal{A})$ (see also [8]).

Lemma 4.4 If the state $x_{T}=\left(\begin{array}{c}y_{T} \\ z_{T}(\cdot)\end{array}\right)$ is reachable from 0 by the system $(2.4)$, then it satisfies the following equivalent conditions:

$$
\begin{aligned}
& \text { (C1) } \sum_{|k|>N} \sum_{m=1}^{n} k^{2}\left|\left\langle\left(\begin{array}{c}
y_{T} \\
z_{T}(\cdot)
\end{array}\right), \psi_{m, k}\right\rangle\right|^{2}<\infty ; \\
& \text { (C2) } \sum_{|k|>N} \sum_{m=1}^{n} k^{2}\left\|P_{m}^{(k)}\left(\begin{array}{c}
y_{T} \\
z_{T}(\cdot)
\end{array}\right)\right\|^{2}<\infty ; \\
& \text { (C3) }\left(\begin{array}{c}
y_{T} \\
z_{T}(\cdot)
\end{array}\right) \in \mathcal{D}(\mathcal{A}) .
\end{aligned}
$$

Let us prove the necessity of the conditions (i) and (ii) of Theorem 1.1 for controllability. 
Theorem 4.5 If the condition (i) of Theorem 1.1 is not verified, i.e. there is $\lambda \in \mathbb{C}$ and $y \in \mathbb{C}^{n} \backslash\{0\}$, such that $\Delta_{\mathcal{A}}^{*}(\lambda) y=0$ and $B^{*} y=0$, then the system (1.1) is not controllable at any time $T>0$.

Proof. The condition (i) may be reformulated as follows: there is no eigenvector $g$ of the operator $\mathcal{A}^{*}$ belonging to Ker $\mathcal{B}^{*}$. This assertion follows from the explicit form (3.13) of eigenvectors of $\mathcal{A}^{*}$.

Assume that there is a vector $g \neq 0$ such that $\mathcal{A}^{*} g=\lambda g$ and $g \in \mathrm{Ker} \mathcal{B}^{*}$. For an arbitrary state $x_{T} \in \mathcal{R}_{T}$ the following equality holds:

$$
\left\langle x_{T}, g\right\rangle=\int_{0}^{T}\left\langle u(t), \mathcal{B}^{*} \mathrm{e}^{\mathcal{A}^{*} t} g\right\rangle \mathrm{d} t=0 .
$$

This means that for any $T>0$ the reachability set $\mathcal{R}_{T}$ is not dense in $M_{2}$ and, therefore, is not equal to $\mathcal{D}(\mathcal{A})$ which is dense in $M_{2}$ since $\mathcal{A}$ is an infinitesimal generator. Thus, the system is not controllable.

Further we show that the controllability of the pair $\left(A_{-1}, B\right)$ is a necessary condition of the controllability of the system (1.1). We prove this assertion in two situations: a singular and a nonsingular matrix $A_{-1}$.

Theorem 4.6 If the condition (ii) of Theorem 1.1 is not verified, i.e. the pair $\left(A_{-1}, B\right)$ is not controllable, then the system (1.1) is also not controllable.

Proof. If the pair $\left(A_{-1}, B\right)$ is not controllable then there is $\mu_{0} \in \sigma\left(A_{-1}\right)$ and $v_{0} \in \mathbb{C}^{n} \backslash\{0\}$ such that $A_{-1}^{*} v_{0}=\overline{\mu_{0}} v_{0}$ and $B^{*} v_{0}=0$.

We begin with the case when $\mu_{0}=0$ is an uncontrollable eigenvalue of $A_{-1}$, i.e.

$$
A_{-1}^{*} v_{0}=0 \text { and } B^{*} v_{0}=0 .
$$

Let us premultiply the equation (1.1) by the vector $v_{0}^{*}$ :

$$
v_{0}^{*} \dot{z}(t)=v_{0}^{*} A_{-1} \dot{z}(t-1)+\int_{-1}^{0}\left[v_{0}^{*} A_{2}(\theta) \dot{z}(t+\theta)+v_{0}^{*} A_{3}(\theta) z(t+\theta)\right] \mathrm{d} \theta+v_{0}^{*} B u .
$$

Taking into account the relations (4.23), we obtain the following equality:

$$
v_{0}^{*} \dot{z}(t)=\int_{-1}^{0}\left[v_{0}^{*} A_{2}(\theta) \dot{z}(t+\theta)+v_{0}^{*} A_{3}(\theta) z(t+\theta)\right] \mathrm{d} \theta .
$$

If we suppose that the system (1.1) is controllable at a time $T>0$ then the set of its solutions under different controls should coincide with the space $H^{1}\left(T-1, T ; \mathbb{C}^{n}\right)$. The latter means that

$$
\left\{v_{0}^{*} \dot{z}(t), t \in[T-1, T]\right\}=L_{2}(T-1, T ; \mathbb{C}) .
$$

On the other hand, the operator $Q(z)=\int_{-1}^{0}\left[v_{0}^{*} A_{2}(\theta) \dot{z}(t+\theta)+v_{0}^{*} A_{3}(\theta) z(t+\theta)\right] \mathrm{d} \theta$, which acts from $H^{1}\left(T-2, T ; \mathbb{C}^{n}\right)$ to $L_{2}(T-1, T ; \mathbb{C})$, is a Fredholm operator. Indeed, changing the time variable $\tau=t+\theta$, we obtain

$$
Q(z)=\int_{t-1}^{t}\left[v_{0}^{*} A_{2}(\tau-t) \dot{z}(\tau)+v_{0}^{*} A_{3}(\tau-t) z(\tau)\right] \mathrm{d} \tau .
$$

Hence, the operator $Q$ is compact and, thus, its image does not coincide with the whole space $L_{2}(T-1, T ; \mathbb{C})$. This contradiction proves the theorem in the case $\mu_{0}=0$. 
Now let us consider the case when only nonzero eigenvalues of $A_{-1}$ are uncontrollable. Without loss of generality we may assume that $\operatorname{det} A_{-1} \neq 0$. Indeed, since $0 \in \sigma\left(A_{-1}\right)$ is a controllable eigenvalue, there is a matrix $P$ such that the matrix $A_{-1}+B P$ is nonsingular (see [23]). Obviously, the pair $\left(A_{-1}+B P, B\right)$ remains uncontrollable. Then, using a change in control, we obtain an equivalent controllability problem for the system with the neutral nonsingular matrix $A_{-1}+B P$.

Since $A_{-1}$ is nonsingular, then the moment equalities (3.17)-(3.18) hold. Consider an uncontrollable eigenvalue $\mu_{m_{0}}$ of $A_{-1}\left(A_{-1}^{*} v_{0}=\bar{\mu}_{m_{0}} v_{0}, B^{*} v_{0}=0, v_{0} \neq 0\right)$ and the subset of (3.17) which corresponds to $m=m_{0}$ :

$$
s_{k}=k\left\langle x_{T}, \psi_{m_{0}, k}\right\rangle=\sum_{d=1}^{r} \int_{0}^{T} \mathrm{e}^{\lambda_{m_{0}}^{k} t} q_{m_{0}, k}^{d} u_{d}(t) \mathrm{d} t, \quad|k|>N,
$$

where $q_{m_{0}, k}^{d}=k\left\langle\mathbf{b}_{d}, \psi_{m_{0}, k}\right\rangle_{M_{2}}$. Let us show that there are sequences $\left\{s_{k}\right\} \in \ell_{2}$ for which the moment problem (4.25) is unsolvable.

For $m=m_{0}$, the eigenvectors of $\widetilde{\mathcal{A}}$ are of the form $\widetilde{\psi}_{m_{0}, k}=\left(v_{0}, \overline{\lambda_{m_{0}}^{k}} \mathrm{e}^{-\overline{\lambda_{m_{0}}^{k}} \theta} v_{0}\right)^{T}$, which implies that $\left\langle\mathbf{b}_{d}, \widetilde{\psi}_{m_{0}, k}\right\rangle_{M_{2}}=\left\langle b_{d}, v_{0}\right\rangle_{\mathbb{C}^{n}}=0$ for all $d=1, \ldots, r$ and $|k|>N$. Applying Lemma 3.2, we obtain the following estimate:

$$
\sum_{|k|>N} k^{2} \sum_{d=1}^{r} \int_{0}^{T}\left|\mathrm{e}^{\lambda_{m}^{k} t}\left\langle\mathbf{b}_{d}, \psi_{m, k}\right\rangle_{M_{2}}\right|^{2} \mathrm{~d} t<+\infty .
$$

It follows from Lemma 4.3 that the solvability set for the system (4.25) is a nontrivial linear manifold $\ell_{T} \subset \ell_{2}, \ell_{T} \neq \ell_{2}$ for any time $T>0$. In other words, there are sequences $\left\{s_{k}\right\}_{|k|>N}$ for which the system of equalities (4.25) is not solvable. This means that there are states $x_{T}$ that satisfy the condition (C1), but which are not reachable from 0 by virtue of the system (1.1). Thus, $\mathcal{R}_{T} \neq \mathcal{D}(\mathcal{A})$ for any $T>0$. This contradiction completes the proof of the theorem.

\section{Sufficiency in the case of one-dimensional control}

In the case of systems with one-dimensional control $\left(r=1, B=b \in \mathbb{C}^{n \times 1}\right)$ the moment problem (3.17)-(3.18) takes the following form:

$$
\begin{aligned}
\alpha_{m, k}\left\langle x_{T}, \psi_{m, k}\right\rangle & =\int_{0}^{T} \mathrm{e}^{\lambda_{m}^{k} t} u(t) \mathrm{d} t, \quad|k|>N, m=1, \ldots, n, \\
\left\langle x_{T}, \widehat{\psi}_{s}\right\rangle & =\int_{0}^{T} \mathrm{e}^{\widehat{\lambda}_{s} t} \widehat{q}_{s}(t) u(t) \mathrm{d} t, \quad s=1, \ldots, \ell_{N},
\end{aligned}
$$

where $N$ is big enough, the family (5.27) is infinite, $\widehat{q}_{j}$ are polynomials, the family (5.28) is finite, and $\alpha_{m, k}=\left(\left\langle\mathbf{b}, \psi_{m, k}\right\rangle_{M_{2}}\right)^{-1}, \mathbf{b}=(b, 0)^{T}$.

From Lemma 3.1 and the explicit form of the basis $\{\psi\}$ of the operator $\mathcal{A}^{*}$ it follows that for all $m=1, \ldots, n$ and $k:|k|>N$ the following estimate holds:

$$
0<C_{1} \leq\left|\frac{1}{k} \alpha_{m, k}\right| \leq C_{2}<+\infty
$$

Our next objective is to find the conditions for the families $\left\{\mathrm{e}^{\lambda_{m}^{k} t}\right\}$ and $\left\{\mathrm{e}^{\widehat{\lambda}_{s} t} \widehat{q}_{s}(t)\right\}$ to form a Riesz basis of the closure of its linear span. 
Let $\delta_{1}, \ldots, \delta_{n}$ be different, modulus $2 \pi \mathrm{i}$, complex numbers, and let $N \in \mathbb{N}$ be natural integer, and let the set $\left\{\varepsilon_{m, k},|k|>N, m=1, \ldots, n\right\} \subset \mathbb{C}^{n}$ be such that $\sum_{m, k}\left|\varepsilon_{m, k}\right|^{2}<+\infty$. We denote by $\mathcal{E}_{N}$ the following (infinite) family of functions:

$$
\mathcal{E}_{N}=\left\{\mathrm{e}^{\left(\delta_{m}+2 \pi \mathrm{i} k+\varepsilon_{m, k}\right) t}, \quad|k|>N, m=1, \ldots, n\right\} .
$$

Next, let $\varepsilon_{1}, \ldots, \varepsilon_{r}$ be another collection of different complex numbers such that $\varepsilon_{j} \neq \delta_{m}+$ $2 \pi \mathrm{i} k+\varepsilon_{m, k}, j=1, \ldots, r, m=1, \ldots, n,|k|>N$, and let $m_{1}^{\prime}, \ldots, m_{r}^{\prime}$ be some positive integers. Let us denote by $\mathcal{E}_{0}$ the following (finite) family of functions

$$
\mathcal{E}_{0}=\left\{\mathrm{e}^{\varepsilon_{j} t}, t \mathrm{e}^{\varepsilon_{j} t}, \ldots, t^{m_{j}^{\prime}-1} \mathrm{e}^{\varepsilon_{j} t}\right\}_{j=1, \ldots, r} .
$$

and by $\mathcal{E}$ the set of functions $\mathcal{E}=\mathcal{E}_{N} \cup \mathcal{E}_{0}$.

Theorem 5.1 (i) If $\sum_{j=1}^{r} m_{j}^{\prime}=(2 N+1) n$, then the family $\mathcal{E}$ forms a Riesz basis in $L_{2}(0, n)$.

(ii) If $T>n$, then independently of the number of elements in $\mathcal{E}_{0}$ the family $\mathcal{E}$ forms a Riesz basis of the closure of its linear span in the space $L_{2}(0, T)$.

The proof of this theorem, based on the results of [1], may be found in [14].

Let us prove the sufficiency of the controllability conditions (i) and (ii) of Theorem 1.1.

Theorem 5.2 Let $u \in \mathbb{C}(r=1)$ and the conditions (i) and (ii) of Theorem 1.1 hold. Then

(1) the system (1.1) is controllable at any time $T>n$;

(2) the estimation of the critical time of controllability is exact, i.e. the system (1.1) is uncontrollable at any time $T \leq n$.

Proof. Let us note that the dimensions of all the eigenspaces (corresponding to different eigenvalues) of $\mathcal{A}^{*}$ are equal to 1 . Indeed, otherwise there is an eigenvector $g$ of the operator $\mathcal{A}^{*}$, such that $\langle\mathbf{b}, g\rangle_{M_{2}}=0$. Since $g=(y, z(\theta))^{T}$, where $y: \Delta_{\mathcal{A}}^{*}\left(\lambda_{0}\right) y=0, \lambda_{0} \in \sigma\left(\mathcal{A}^{*}\right)$, and since $\langle\mathbf{b}, g\rangle_{M_{2}}=\langle b, y\rangle_{\mathbb{C}^{n}}$, we obtain a contradiction with condition (i).

Let us consider the problem (5.27)-(5.28). From condition (i) it follows that $\left\langle\mathbf{b}, \psi_{m, k}\right\rangle_{M_{2}} \neq$ 0 for all $m$ and $k$. Moreover, all polynomial $\left\{\widehat{q}_{s}(t)\right\}, s=1, \ldots, \ell_{N}$ are nontrivial. By the moment problem we construct the following families of functions:

$$
\begin{aligned}
& \Phi_{1}=\left\{\mathrm{e}^{\lambda_{m}^{k} t},|k|>N, m=1, \ldots, n\right\}, \\
& \Phi_{2}=\left\{\mathrm{e}^{\widehat{\lambda}_{s} t} \widehat{q}_{s}(t), s=1, \ldots, \ell_{N}\right\} .
\end{aligned}
$$

Due to Theorem 5.1 for $T>n$ and for big enough $N$, the family

$$
\Phi=\Phi_{1} \bigcup \Phi_{2}
$$

constitutes a Riesz basis in ClLin $\Phi \subset L_{2}(0, T)$. Thus, due to Lemma 4.1, the moment problem (5.27)-(5.28) is solvable if and only if the right-hand side is an element of $\ell_{2}$, or, equivalently the condition (C1) from Lemma 4.4 holds. Since (C1) is equivalent to (C3) we conclude that for $T>n$ the moment problem is solvable if and only if $x_{T} \in \mathcal{D}(\mathcal{A})$, i.e. $\mathcal{R}_{T}=\mathcal{D}(\mathcal{A})$.

To prove assertion (2) we recall that the number of elements in family $\Phi_{2}$ is equal to $\ell_{N}=(2 N+2) n$. On the other hand, it follows from Theorem 5.1 that in $L_{2}(0, n)$ one has

$$
\operatorname{codim} \mathrm{Cl} \operatorname{Lin} \Phi_{1}=(2 N+1) n .
$$


Thus, the family $\Phi=\Phi_{1} \cup \Phi_{2}$ contains $n$ functions, which can be represented as linear combination of other functions from this family. This means that the codimension $\mathcal{R}_{T}$ in $\mathcal{D}(\mathcal{A})$ is not equal to zero: $\operatorname{codim} \mathcal{R}_{T}=n$. Hence, the reachability set $\mathcal{R}_{T}$ for $T=n$ is not equal to $\mathcal{D}(\mathcal{A})$ and the system is not controllable. For $T<n$ it follows from Lemma 4.2 that the codimension of the set $\mathcal{R}_{T}$ in $\mathcal{D}(\mathcal{A})$ is infinite.

\section{Sufficient conditions: the multivariable case}

Consider the case $\operatorname{dim} B=r>1$. Without loss of generality we assume that the pair $\left(A_{-1}, B\right)$ is in the Frobenius normal form, i.e. $A_{-1}=\operatorname{diag}\left\{F_{1}, \ldots, F_{r}\right\}, \operatorname{dim} F_{i}=s_{i}$, and $F_{i}$ are of the form $(2.9) ; B=\operatorname{diag}\left\{g_{1}, \ldots, g_{r}\right\}$, where $g_{i}=(0,0, \ldots, 1)^{\mathrm{T}} \in \mathbb{C}^{s_{i}}$. It is well-known that

$$
\max _{i} \operatorname{dim} F_{i}=n_{1}
$$

where $n_{1}$ is the first controllability index of the pair $\left(A_{-1}, B\right)$, i.e. $n_{1}$ is the minimal integer $\nu$ satisfying the relation $\operatorname{rank}\left(B, A_{-1} B, \ldots, A_{-1}^{\nu-1} B\right)=n$.

According to the representation in the Frobenius form, we rewrite the infinite part (3.17) of the moment problem as follows:

$$
\begin{aligned}
& k\left\langle x_{T}, \psi_{m, k}\right\rangle=\int_{0}^{T} \mathrm{e}^{\lambda_{m}^{k} t} q_{m, k}^{1} u_{1}(t) \mathrm{d} t+\sum_{d \neq 1} \int_{0}^{T} \mathrm{e}^{\lambda_{m}^{k} t} q_{m, k}^{d} u_{d}(t) \mathrm{d} t, \quad m \in S_{1}, \\
& k\left\langle x_{T}, \psi_{m, k}\right\rangle=\int_{0}^{T} \mathrm{e}^{\lambda_{m}^{k} t} q_{m, k}^{2} u_{2}(t) \mathrm{d} t+\sum_{d \neq 2} \int_{0}^{T} \mathrm{e}^{\lambda_{m}^{k} t} q_{m, k}^{d} u_{d}(t) \mathrm{d} t, \quad m \in S_{2}, \\
& k\left\langle x_{T}, \psi_{m, k}\right\rangle=\int_{0}^{T} \mathrm{e}^{\lambda_{m}^{k} t} q_{m, k}^{r} u_{r}(t) \mathrm{d} t+\sum_{d \neq r} \int_{0}^{T} \mathrm{e}^{\lambda_{m}^{k} t} q_{m, k}^{d} u_{d}(t) \mathrm{d} t, \quad m \in S_{r},
\end{aligned}
$$

where $S_{1}=\left\{1, \ldots, s_{1}\right\}, S_{2}=\left\{s_{1}+1, \ldots, s_{1}+s_{2}\right\}, \ldots, S_{r}=\left\{s_{1}+\ldots, s_{r-1}+1, \ldots, n\right\}$.

Next we apply Theorem 5.1 to the family of functions from (6.30). Let us fix $d \in\{1, \ldots, r\}$ and choose an arbitrary subset of $L \subset\{1, \ldots, n\}$.

Theorem 6.1 For arbitrary $d, L$, and for all $T>n^{\prime}=|L|$ the set

$$
\Phi_{1}=\left\{\mathrm{e}^{\lambda_{m}^{k} t} q_{m, k}^{d}, \quad|k|>N ; m \in L\right\}
$$

constitutes a Riesz basis of the closure of its linear span $\mathrm{Cl} \operatorname{Lin} \Phi_{1}$ in $L_{2}(0, T)$.

If $T=n^{\prime}$, then codim $\mathrm{Cl} \operatorname{Lin} \Phi_{1}=(2 N+1) n^{\prime}$ in the space $L_{2}\left(0, n^{\prime}\right)$.

Proof. Let us consider the linear operator $\mathcal{T}: \operatorname{Lin} \Phi_{1} \rightarrow \operatorname{Lin} \Phi_{1}$ defined on elements of $\Phi_{1}$ by the following relations

$$
\mathcal{T}\left(\mathrm{e}^{\lambda_{m}^{k} t} q_{m, k}^{d}\right)=\mathrm{e}^{\lambda_{m}^{k} t}, \quad|k|>N, m \in L .
$$

Due to Lemma 3.1 the family $\left\{q_{m, k}^{d}\right\}$ is uniformly bounded. Thus, from Theorem 5.1 we obtain that the operator $\mathcal{T}$ is bounded in the sense of $L_{2}(0, T)$ and its extension to $L=\mathrm{Cl} \operatorname{Lin} \Phi_{1}$ is a bounded one-to-one operator from $L$ to $L$.

Hence, since due to Theorem 5.1 the images of the elements of $\Phi_{1}$ form a Riesz basis of $L$, then $\Phi_{1}$ is also a Riesz basis of $L$ in $L_{2}(0, T)$.

We also need the following result (see [14, Theorem 5.5]). 
Theorem 6.2 Consider the system (2.4) and suppose that there is an integer $N$ and a time $T_{0}>0$ such that the moment problem (3.17) is solvable for $T=T_{0}$ and for all sequences $\left\{k\left\langle x_{T}, \psi_{m, k}\right\rangle\right\}_{|k|>N}$, satisfying $(\mathrm{C} 1)$.

Then, from condition (i) of Theorem 1.1 it follows that $\mathcal{R}_{T}=\mathcal{D}(\mathcal{A})$ for $T>T_{0}$.

Now we prove the main result of this section.

Theorem 6.3 Let the conditions (i) and (ii) of Theorem 1.1 hold for a system of the form (1.1). Then the system (1.1) is controllable and, moreover, the critical time of controllability is $T_{0}=$ $n_{1}$, where $n_{1}$ is the first controllability index of the pair $\left(A_{-1}, B\right)$.

Proof. We assume that the pair $\left(A_{-1}, B\right)$ is in the Frobenius normal form. Then for all $i=1, \ldots, r, m \in S_{i}, d \neq i$ and for all $|k|>N$ the following relation holds:

$$
\left\langle\mathbf{b}_{d}, \widetilde{\psi}_{m, k}\right\rangle_{M_{2}}=\left\langle b_{d}, c_{m}\right\rangle_{\mathbb{C}^{n}}=0
$$

where $c_{m}: A_{-1} c_{m}=\mu_{m} c_{m}$. Thus for all $i=1, \ldots, r$ and $m \in S_{i}$ the following equality holds

$$
\sum_{d \neq i} \int_{0}^{T} \mathrm{e}^{\lambda_{m}^{k} t} q_{m, k}^{d} u_{d}(t) \mathrm{d} t=\sum_{d \neq i} \int_{0}^{T} k\left(\mathrm{e}^{\lambda_{m}^{k} t}\left\langle\mathbf{b}_{d}, \psi_{m, k}\right\rangle_{M_{2}}-\mathrm{e}^{\widetilde{\lambda}_{m}^{k} t}\left\langle\mathbf{b}_{d}, \widetilde{\psi}_{m, k}\right\rangle_{M_{2}}\right) u_{d}(t) \mathrm{d} t .
$$

For any $N \in \mathbb{N}$ the moment problem (6.30) may be written in operator form

$$
\left\{S_{m, k}\right\}=Z_{N} u(\cdot)+Q_{N} u(\cdot),
$$

where $\left\{S_{m, k}\right\}=\left\{k\left\langle x_{T}, \psi_{m, k}\right\rangle\right\}$ and the operators $Z_{N}, Q_{N}: L_{2}\left(0, T ; \mathbb{C}^{r}\right) \rightarrow \ell_{2}$ are of the form

$$
\begin{aligned}
& Z_{N} u(\cdot)=\left\{\int_{0}^{T} \mathrm{e}^{\lambda_{m}^{k} t} q_{m, k}^{i} u_{i}(t) \mathrm{d} t,|k|>N\right\}, \\
& Q_{N} u(\cdot)=\left\{\sum_{d \neq i} \int_{0}^{T} k\left(\mathrm{e}^{\lambda_{m}^{k} t}\left\langle\mathbf{b}_{d}, \psi_{m, k}\right\rangle_{M_{2}}-\mathrm{e}^{\widetilde{\lambda}_{m}^{k} t}\left\langle\mathbf{b}_{d}, \widetilde{\psi}_{m, k}\right\rangle_{M_{2}}\right) u_{d}(t) \mathrm{d} t,|k|>N\right\} .
\end{aligned}
$$

Due to Theorem 6.1, for a big enough $N$ and for $T \geq n_{1}$, the operator $Z_{N}$ is surjective, i.e. its image of the space $L_{2}\left(0, T ; \mathbb{C}^{r}\right)$ is the whole space $\ell_{2}$. From Lemma 3.2, it follows that for big enough $N$ the operator $Q_{N}$ is compact, and, moreover, $\left\|Q_{N}\right\| \rightarrow 0$ when $N \rightarrow+\infty$.

Let us show that there is $N_{0} \in \mathbb{N}$ such that for all $N>N_{0}$ one has:

$$
\operatorname{Im}\left[Z_{N}+Q_{N}\right]=\ell_{2}
$$

Since $\operatorname{Im} Z_{N}=\ell_{2}$, then there is a constant $\gamma_{N}>0$ such that $\left\|Z_{N}^{*} x\right\| \geq \gamma_{N}\|x\|$ for all $x \in \ell_{2}$ (see, e.g. [21, Theorem 4.13]). For $N>N_{0}$ we introduce the notation $\ell_{2}^{N}=\left\{\left\{S_{m, k}\right\}_{|k|>N}\right.$ : $\left.\sum_{|k|>N}\left|s_{k}\right|^{2}<+\infty\right\}$. Thus, we have $Z_{N}=P Z_{N_{0}}$, where projectors $P: \ell_{2}^{N_{0}} \rightarrow \ell_{2}^{N}$ are defined as follows:

$$
P\left(\left\{S_{m, k}\right\}_{|k|>N_{0}}\right)=\left\{S_{m, k}\right\}_{|k|>N} .
$$

Therefore, $Z_{N}^{*}=Z_{N_{0}}^{*} P^{*}$ and $\left\|P^{*} x\right\|=\|x\|$, which gives

$$
\left\|Z_{N}^{*} x\right\|=\left\|Z_{N_{0}}^{*} P^{*} x\right\| \geq \gamma_{N_{0}}\|x\| .
$$

The latter means that for all $N>N_{0}$ and $x \in \ell_{2}$ the inequality $\left\|Z_{N}^{*} x\right\| \geq \gamma\|x\|$ holds, where $\gamma=\gamma_{0}$. Since $\left\|Q_{N}\right\| \rightarrow 0$ when $N \rightarrow+\infty$, then by choosing an appropriate $N$ we obtain the estimate $\left\|Z_{N}-\left(Z_{N}+Q_{N}\right)\right\|=\left\|Q_{N}\right\| \leq \frac{\gamma}{2}$. Thus

$$
\left\|\left[Z_{N}^{*}+Q_{N}^{*}\right] x\right\| \geq\left\|Z_{N}^{*} x\right\|-\left\|Q_{N}^{*} x\right\| \geq \gamma\|x\|-\frac{\gamma}{2}\|x\|=\frac{\gamma}{2}\|x\| .
$$


Therefore, the operator $Z_{N}+Q_{N}$ is surjective and its image is equal to $\ell_{2}$.

Thus, the moment problem (6.30) is solvable for $T \geq n_{1}$ and big enough $N \in \mathbb{N}$. Applying Theorem 6.2, we obtain that $R_{T}=\mathcal{D}(\mathcal{A})$ for $T>n_{1}$.

Arguing as in the proof of Theorem 5.2, we show that the codimension $\mathcal{R}_{n_{1}}$ in $\mathcal{D}(\mathcal{A})$ is finite and no less than $n_{1}$, which means that the system (1.1) is uncontrollable at time $T=n_{1}$. For $T<n_{1}$, the codimension of $\mathcal{R}_{T}$ in $\mathcal{D}(\mathcal{A})$ is infinite.

\section{$7 \quad$ Example}

Consider a three-dimensional $(n=3)$ system given by the equation (1.1) with the following coefficients:

$$
A_{-1}=\left(\begin{array}{rrr}
-4 & 6 & -4 \\
0 & 2 & -2 \\
-3 & 3 & 2
\end{array}\right), \quad B=\left(\begin{array}{rr}
1 & 1 \\
1 & 0 \\
-1 & 1
\end{array}\right)
$$

and the matrices $A_{2}(\theta), A_{3}(\theta)$ are such that $\operatorname{rank}\left(\Delta_{\mathcal{A}}(\lambda) B\right)=n$ for all $\lambda \in \mathbb{C}$.

We apply the change in control and state variables $u(t)=P \dot{z}(t-1)+v(t), w=C z$, where

$$
P=\left(\begin{array}{lll}
1 & -1 & 2 \\
3 & -2 & 3
\end{array}\right), \quad C=\left(\begin{array}{rrr}
1 & -1 & 1 \\
1 & 1 & 0 \\
-1 & 0 & 1
\end{array}\right)
$$

and obtain the following system:

$$
\dot{w}(t)=\widehat{A}_{-1} \dot{w}(t-1)+\int_{-1}^{0} \widehat{A}_{2}(\theta) \dot{w}(t+\theta) \mathrm{d} \theta+\int_{-1}^{0} \widehat{A}_{3}(\theta) w(t+\theta) \mathrm{d} \theta+\widehat{B} v,
$$

where $\widehat{A}_{-1}$ and $\widehat{B}$ are of the form

$$
\widehat{A}_{-1}=\left(\begin{array}{lll}
2 & 0 & 0 \\
0 & 0 & 1 \\
0 & 3 & 2
\end{array}\right), \quad \widehat{B}=\left(\begin{array}{ll}
1 & 0 \\
0 & 0 \\
0 & 1
\end{array}\right)=\left(b_{1}, b_{2}\right) .
$$

Let the operator $\mathcal{A}$ with eigenvalues $\lambda_{m}^{k}$ corresponds to the perturbed system (7.33)-(7.34), and the operator $\widetilde{\mathcal{A}}$ with eigenvalues $\widetilde{\lambda}_{m}^{k}$ corresponds to the system $\dot{w}(t)=\widehat{A}_{-1} \dot{w}(t-1)$. Since the pair $\left(\widehat{A}_{-1}, \widehat{B}\right)$ is in the Frobenius normal form, then the eigenvectors $\widetilde{\psi}_{m, k}$ of the operator $\widetilde{\mathcal{A}}^{*}$ satisfy the relations

$$
\begin{aligned}
\left\langle\mathbf{b}_{1}, \widetilde{\psi}_{m, k}\right\rangle & =0, \quad m=2,3 \\
\left\langle\mathbf{b}_{2}, \widetilde{\psi}_{m, k}\right\rangle & =0, \quad m=1, \quad \mathbf{b}_{i}=\left(b_{i}, 0\right) \in M_{2} .
\end{aligned}
$$

Since $q_{m, k}^{d}=k\left\langle\mathbf{b}_{d}, \psi_{m, k}\right\rangle$, where $\psi_{m, k}$ are eigenvectors of the operator $\mathcal{A}^{*}$, then the infinite part of the moment problem (3.17) reads as

$$
\begin{aligned}
k\left\langle x_{T}, \psi_{1, k}\right\rangle & =\int_{0}^{T} \mathrm{e}^{\lambda_{1}^{k} t} q_{1, k}^{1} u_{1}(t) \mathrm{d} t+\int_{0}^{T} f_{1, k}^{2}(t) u_{2}(t) \mathrm{d} t, \\
k\left\langle x_{T}, \psi_{2, k}\right\rangle & =\int_{0}^{T} \mathrm{e}^{\lambda_{2}^{k} t} q_{2, k}^{2} u_{2}(t) \mathrm{d} t+\int_{0}^{T} f_{2, k}^{2}(t) u_{1}(t) \mathrm{d} t, \\
k\left\langle x_{T}, \psi_{3, k}\right\rangle & =\int_{0}^{T} \mathrm{e}^{\lambda_{3}^{k} t} q_{3, k}^{2} u_{2}(t) \mathrm{d} t+\int_{0}^{T} f_{3, k}^{2}(t) u_{1}(t) \mathrm{d} t, \quad|k|>N,
\end{aligned}
$$


where the functions $f_{m, k}^{d}$ are of the form

$$
f_{m, k}^{d}(t)=k\left(\mathrm{e}^{\lambda_{m}^{k} t}\left\langle\mathbf{b}_{d}, \psi_{m, k}\right\rangle-\mathrm{e}^{\tilde{\lambda}_{m}^{k} t}\left\langle\mathbf{b}_{d}, \widetilde{\psi}_{m, k}\right\rangle\right)
$$

and, due to Lemma 3.2, they satisfy the estimate $\left|f_{m, k}^{d}(t)\right| \leq \alpha_{k}, \sum_{k} \alpha_{k}^{2}<+\infty$.

The first controllability index $n_{1}$ of the pair $\left(\widehat{A}_{-1}, \widehat{B}\right)$ (or $\left(A_{-1}, B\right)$ ) equals 2 . The conditions (i) and (ii) of Theorem 1.1 are satisfied and thus, the system is controllable with the critical controllability time $T_{0}=2$.

\section{Conclusion}

A new approach to the problem of exact controllability by the moment problem method is proposed. The difficulty of the choosing the basis is overcome by a change in the control and phase coordinates, which enables a more direct proof of the criterion of exact controllability. The proposed approach offers a new challenge for controllability and stabilizability problems for a more general class of systems with a neutral operator of the form $K f=\sum_{i=1}^{r} A_{h_{i}} f\left(h_{i}\right)$, $h_{i} \in[-1,0]$.

\section{Acknowledgements}

This research was partially supported by PROMEP (Mexico) via "Poyecto de Redes" and the Polish Nat. Sci. Center, grant No 514238438.

\section{References}

[1] S. A. Avdonin and S. A. Ivanov. Families of exponentials. Cambridge University Press, Cambridge, 1995.

[2] H. T. Banks, Marc Q. Jacobs, and C. E. Langenhop. Characterization of the controlled states in $W_{2}^{(1)}$ of linear hereditary systems. SIAM J. Control, 13:611-649, 1975.

[3] Z. Bartosiewicz. A criterion of closedness of an attainable set of a delay system. Systems Control Lett., 3(4):211-215, 1983.

[4] A. Bensoussan, G. Da Prato, M. C. Delfour, and S. K. Mitter. Representation and control of infinite-dimensional systems. Vol. 1. Birkhäuser, Boston, MA, 1992.

[5] J. A. Burns, T. L. Herdman, and H. W. Stech. Linear functional-differential equations as semigroups on product spaces. SIAM J. Math. Anal., 14(1):98-116, 1983.

[6] R. Gabasov and Kirillova, F. Kachestvennaya teoriya optimalnykh protsessov. Izdat. "Nauka", Moscow, 1971. Monographs in Theoretical Foundations of Technical Cybernetics.

[7] I. C. Gohberg and M. G. Krein. Introduction to the theory of linear nonselfadjoint operators. Translations of Mathematical Monographs, Vol. 18. American Mathematical Society, Providence, R.I., 1969.

[8] K. Ito and T. J. Tarn. A linear quadratic optimal control for neutral systems. Nonlinear Anal., 9(7):699-727, 1985. 
[9] M. Q. Jacobs and C. E. Langenhop. Criteria for function space controllability of linear neutral systems. SIAM J. Control Optimization, 14(6):1009-1048, 1976.

[10] V. E. Khartovskiı and A. T. Pavlovskaya. Complete controllability and controllability for linear autonomous systems of neutral type. Automation and Remote Control, (5):769-784, 2013.

[11] A. Manitius and R. Triggiani. Function space controllability of linear retarded systems: a derivation from abstract operator conditions. SIAM J. Control Optim., 16(4):599-645, 1978.

[12] V. M. Marčenko. On the controllability of zero function of time lag systems. Problems Control Inform. Theory/Problemy Upravlen. Teor. Inform., 8(5-6):421-432, 1979.

[13] D. A. O'Connor and T. J. Tarn. On the function space controllability of linear neutral systems. SIAM J. Control Optim., 21(2):306-329, 1983.

[14] R. Rabah and G. M. Sklyar. The analysis of exact controllability of neutral-type systems by the moment problem approach. SIAM J. Control Optim., 46(6):2148-2181, 2007.

[15] R. Rabah, G. M. Sklyar, and A. V. Rezounenko. Generalized Riesz basis property in the analysis of neutral type systems. C. R. Math. Acad. Sci. Paris, 337(1):19-24, 2003.

[16] R. Rabah, G. M. Sklyar, and A. V. Rezounenko. Stability analysis of neutral type systems in Hilbert space. J. Differential Equations, 214(2):391-428, 2005.

[17] R. Rabah, G. M. Sklyar, and A. V. Rezounenko. On strong regular stabilizability for linear neutral type systems. J. Differential Equations, 245(3):569-593, 2008.

[18] R. Rabah and G. Sklyar. On exact controllability of linear time delay systems of neutral type. In Applications of time delay systems, volume 352 of Lecture Notes in Control and Inform. Sci., pages 165-171. Springer, Berlin, 2007.

[19] R. Rabah, G. M. Sklyar, and P. Yu Barkhayev. Stability and stabilizability of mixed retarded-neutral type systems. ESAIM Control Optim. Calc. Var., 18(3):656-692, 2012.

[20] H. Rivera Rodas and C. E. Langenhop. A sufficient condition for function space controllability of a linear neutral system. SIAM J. Control Optim., 16(3):429-435, 1978.

[21] W. Rudin. Functional analysis. International Series in Pure and Applied Mathematics. McGraw-Hill Inc., New York, second edition, 1991.

[22] B. Shklyar. Exact null controllability of abstract differential equations by finite-dimensional control and strongly minimal families of exponentials. Diff. Equ. Appl., 3(2):171-188, 2011.

[23] W. M. Wonham, Linear multivariable control: a geometric approach. Springer, New York, 3rd Edition, 1985.

[24] Robert M. Young. An introduction to nonharmonic Fourier series. Academic Press, New York, 1980. 